\title{
Common Fixed Point Result of Multivalued and Singlevalued Mappings in Partially Ordered Metric Space
}

\author{
Rajesh Kumar Saini, Archana Sharma \\ Department of Mathematical Sciences and Computer Applications, Bundelkhand University, Jhansi, India \\ Email: rksaini03@yahoo.com
}

Received July 19, 2012; revised September 28, 2012; accepted October 8, 2012

\begin{abstract}
In recent times the fixed point resulting in partially ordered metric spaces has greatly developed. In this paper we prove common fixed point results for multivalued and singlevalued mappings in partially ordered metric space. Our theorems generalized the theorem in [1] and extended much more recent results in such spaces.
\end{abstract}

Keywords: Multi-Valued Mapping; Single-Valued Mapping; Partial Ordering; Control Function; Fixed Point Theorem

\section{Introduction and Preliminaries}

Throughout this paper, let $(X, d)$ be a metric space unless mentioned otherwise and $B(X)$ is the set of all non-empty bounded subsets of $X$. Let $\delta(A, B)$ and $D(A, B)$ be the functions defined by

$$
\begin{aligned}
& \delta(A, B)=\sup \{d(a, b): a \in A, b \in B\} \\
& D(A, B)=\inf \{d(a, b): a \in A, b \in B\}
\end{aligned}
$$

for all $A, B$ in $B(X)$. If $A$ is a singleton i.e. $A=\{a\}$, we write

$$
\delta(A, B)=\delta(a, B)
$$

and

$$
D(A, B)=D(a, B)
$$

If $B$ is also a singleton i.e. $B=\{b\}$, we write

$$
\delta(A, B)=\delta(A, b)
$$

and

$$
D(A, B)=D(A, b)
$$

It is obvious that $D(A, B)=\delta(a, B)$. For all $A, B, C \in$ $B(X)$. The definition of $d(A, B)$ yields the following:

$$
\begin{gathered}
\delta(A, B)=\delta(B, A) \leq 0 \\
\delta(A, B) \leq \delta(A, C)+\delta(C, B) \\
\delta(A, B)=0 \text { iff } A=B=\{a\}
\end{gathered}
$$

and

$$
\delta(A, A)=\operatorname{diam}\{a\} .
$$

Several authors used these concepts of weakly contraction, compatibility, weak compatibility to prove some common fixed point theorems for set valued mappings (see [2-8]).

Definition 1.1. [9] $A$ sequence $\left\{A_{n}\right\}$ of subsets of $X$ is said to be convergent to a subset $A$ of $X$ if

1) Given $a \in A$, there is a sequence $\left\{a_{n}\right\}$ in $X$ such that $a_{n} \in A_{n}$ for $n=1,2, \cdots$, and $\left\{a_{n}\right\}$ converges to $a$.

2) Given $\varepsilon>0$, there exists a positive integer $N$ such that $A_{n} \subseteq A_{\varepsilon}$ for $n>N$ where $A_{e}$ is the union of all open spheres with centers in $A$ and radius $\varepsilon$.

Lemma 1.1. $[9,10]$ If $\left\{A_{n}\right\}$ and $\left\{B_{n}\right\}$ are sequences in $B(X)$ converging to $A$ and $B$ in $B(X)$, respectively, then the sequence $\left\{\delta\left(A_{n}, B_{n}\right)\right\}$ converges to $\delta(A, B)$.

Lemma 1.2. [9] Let $\left\{A_{n}\right\}$ be a sequence in $B(X)$ and $y$ a point in $X$ such that $\delta\left(A_{n}, y\right) \rightarrow 0$. Then the sequence $\left\{A_{n}\right\}$ converges to the set $\{y\}$ in $B(X)$.

In [11], Jungck and Rhoades extended definition of compatibility to set valued mappings setting as follows:

Definition 1.2. The mapping $I: X \rightarrow X$ and $f: X \rightarrow B(X)$ are $\delta$-compatible if $\lim _{n \rightarrow \infty} \delta\left(f I x_{n}, I f x_{n}\right)=0$, whenever $\left\{x_{n}\right\}$ is a sequence in $X$ such the If $x_{n} \in B(X), f x_{n} \rightarrow\{t\}$ and $I x_{n} \rightarrow t$, for some $t \in X$.

Recently, the following definition is given by Jungck and Rhoades [12].

Definition 1.3. The mapping $I: X \rightarrow X$ and $f: X \rightarrow B(X)$ are weakly compatible if for each point $u$ in $X$ such that $f u=\{I u\}$, we have $f I u=I f u$.

It can be seen that any $\delta$-compatible mappings are 
weakly compatible but the converse is not true as shown by an example in [13]. We will use the following relation between two nonempty subsets of a partially ordered set.

Definition 1.4. [3] Let $A$ and $B$ be two nonempty subsets of a partially ordered set $(X ; \preccurlyeq)$. The relation between $A$ and $B$ is denoted and defined as follows: $A \preccurlyeq B$, if for every $a \in A$ there exists $b \in B$ such that $a \preccurlyeq b$.

We will utilize the following control function which is also referred to as altering distance function.

Definition 1.5. [14] A function $\psi:[0, \infty) \rightarrow[0, \infty)$ is called an Altering distance function if the following properties are satisfied:

1) $\psi$ is monotone increasing and continuous,

2) $\psi(t)=0$ if and only if $t=0$.

For the use of control function in metric fixed point theory see some recent references $([15,16])$.

\section{Main Result}

Recently fixed point theory in partially ordered metric spaces has greatly developed. Choudhury and Metiya [17] proved certain fixed point theorems for multi valued and single valued mappings in partially ordered metric spaces. They proved the following:

Theorem 2.1. Let $(X, \preccurlyeq)$ be a partially ordered set and suppose that there exists a metric $d$ on $X$ such that $(X, d)$ is a complete metric space. Let $T: X \rightarrow B(X)$ be a multi valued mappings such that the following conditions are satisfied:

There exists $x_{0} \in X$ such that $\left\{x_{0}\right\} \prec_{1} T x_{0}$,

1) For $x, y \in X, x \prec_{1} y$ implies $T x \prec_{1} T y$,

2) If $x_{n} \rightarrow x$ is a non decreasing sequence in $X$, then $x_{n} \preccurlyeq x$, for all $n$,

3) $\psi(d(T x, T y)) \leq \alpha \psi\left(\max \left\{d(x, y), D(x, T x), D(y, T y), \frac{D(x, T y)+D(y, T x)}{2}\right\}\right)$,

for all comparable $x, y \in X$, where $0<\alpha<1$ and $\psi$ is an Altering distance function. Then $T$ has a fixed point.

We prove the following theorem for four single-valued and multivalued mappings:

Theorem 2.2. Let $(X, \preccurlyeq)$ be a partially ordered set and suppose that there exists a metric $d$ on $X$ such that $(X, d)$ is a complete metric space. Let $I, J: X \rightarrow X$ be single valued and $F, G: X \rightarrow C B(X)$ be multivalued mappings such that the following conditions are satisfied:

1) $\cup F(X) \prec_{1} J(X)$ and $\cup G(X) \prec_{1} I(X)$,

2) $\{F, I\}$ and $\{G, J\}$ are weakly compatible,

3) If $x_{n} \rightarrow x$ is a strictly decreasing sequence in $X$, then $x_{n} \preccurlyeq x$, for all $n$,

4) $\psi(d(F x, G y)) \leq \alpha \psi\left(\max \left\{d(I x, J y), D(I x, F x), D(J y, G y), \frac{D(I x, G y)+D(I y, F x)}{2}\right\}\right)$,

for all comparable $x, y \in X$, $\operatorname{diam} F x=\operatorname{diam} G y$, where $0<\alpha<1$ and $\psi$ is an Altering distance function and suppose that one of $I(X)$ or $J(X)$ is complete. Then there exists a unique point $p \in X$ such that

$$
F p=G p=\{I p\}=\{J p\}=\{p\}
$$

Proof: Let $x_{0}$ be an arbitrary point of $X$. By 1) we choose a point $x_{1} \in X$ such that $y_{1}=F x_{0} \prec_{1} J x_{1}$. For this point $x_{1}$, there exists a point $x_{2} \in X$ such that $y_{2}=G x_{1} \prec_{1} I x_{2}$, and so on. Continuing in this manner we can define a sequence $\left\{y_{n}\right\}$ as follows

$$
\begin{aligned}
& y_{2 n+1}=F x_{2 n} \prec_{1} J x_{2 n+1}, \\
& y_{2 n+2}=F x_{2 n+1} \prec_{1} J x_{2 n+2},
\end{aligned}
$$

We claim that $\left\{y_{n}\right\}$ is a Cauchy sequence. For which two cases arise, either $y_{n}=y_{n+1}$ for some $n$, or $y_{n} \neq y_{n+1}$, for each $n$.

Case I. If $y_{n}=y_{n+1}$ for some $n$ then, $y_{n}=y_{n+k}$ for each $k \geq 1$. For instance suppose $y_{2 m+1}=y_{2 m+2}$. Then $y_{2 m+2}=y_{2 m+3}$. Otherwise using 3 ), we get

$$
\begin{aligned}
& \psi\left(d\left(y_{2 m+2}, y_{2 m+3}\right)\right)=\psi\left(d\left(F x_{2 m+1}, G x_{2 m+2}\right)\right) \\
& \leq \alpha \psi\left(\max \left\{d\left(I x_{2 m+1}, J x_{2 m+2}\right), D\left(I x_{2 m+1}, F x_{2 m+1}\right), D\left(J x_{2 m+2}, G x_{2 m+2}\right), \frac{D\left(I x_{2 m+1}, G x_{2 m+2}\right)+D\left(I x_{2 m+2}, F x_{2 m+1}\right)}{2}\right\}\right) \\
& \leq \alpha \psi\left(\max \left\{d\left(y_{2 m+1}, y_{2 m+2}\right), d\left(y_{2 m+1}, y_{2 m+2}\right), d\left(y_{2 m+2}, y_{2 m+3}\right), \frac{d\left(y_{2 m+1}, y_{2 m+3}\right)+d\left(y_{2 m+2}, y_{2 m+2}\right)}{2}\right\}\right)
\end{aligned}
$$

Since

$$
\frac{d\left(y_{2 m+1}+y_{2 m+3}\right)}{2} \leq \max \left\{d\left(y_{2 m+1}, y_{2 m+2}\right), d\left(y_{2 m+2}, y_{2 m+3}\right)\right\}
$$


It follows that

$$
\psi\left(d\left(y_{2 m+2}, y_{2 m+3}\right)\right) \leq \alpha \psi\left(\max \left\{d\left(y_{2 m+1}, y_{2 m+2}\right), d\left(y_{2 m+2}, y_{2 m+3}\right)\right\}\right)
$$

Suppose that if $d\left(y_{2 m+1}, y_{2 m+2}\right) \leq d\left(y_{2 m+2}, y_{2 m+3}\right)$, for some positive integer $n$, then from (2.2), we have

$$
\psi\left(d\left(y_{2 m+2}, y_{2 m+3}\right)\right) \leq \alpha \psi\left(d\left(y_{2 m+2}, y_{2 m+3}\right)\right)
$$

which implies that $d\left(y_{2 m+2}, y_{2 m+3}\right)=0$, or $y_{2 m+2}=y_{2 m+3}$.
Hence $y_{2 m+2}=y_{2 m+3}$. Similarly $y_{2 m+3}=y_{2 m+4}$ implie $y_{2 m+4}=y_{2 m+5}$. Proceeding in this manner, it follows that $y_{2 m+1}=y_{2 m+k}$ for each $k>1$, so that $y_{n}=y_{n+k}$ for each $k \geq 1$, for some $n$, and $\left\{y_{n}\right\}$ is a Cauchy sequence.

Case II. When $y_{n} \neq y_{n+1}$ for each $n$. In this case, using 3), we obtain

$$
\begin{aligned}
& \psi\left(d\left(y_{2 n+1}, y_{2 n+2}\right)\right)=\psi\left(d\left(F x_{2 n}, G x_{2 n+1}\right)\right) \\
& \leq \alpha \psi\left(\max \left\{d\left(I x_{2 n}, J x_{2 n+1}\right), D\left(I x_{2 n}, F x_{2 n}\right), D\left(J x_{2 n+1}, G x_{2 n+1}\right), \frac{D\left(I x_{2 n}, G x_{2 n+1}\right)+D\left(I x_{2 n+1}, F x_{2 n}\right)}{2}\right\}\right) \\
& \leq \alpha \psi\left(\max \left\{d\left(y_{2 n}, y_{2 n+1}\right), d\left(y_{2 n}, y_{2 n+1}\right), d\left(y_{2 n+1}, y_{2 n+2}\right), \frac{d\left(y_{2 n+1}, y_{2 n+2}\right)+d\left(y_{2 n+1}, y_{2 n+1}\right)}{2}\right\}\right),
\end{aligned}
$$

Since

It follows that

$$
\frac{d\left(y_{2 n+1}, y_{2 n+2}\right)}{2} \leq \max \left\{d\left(y_{2 n}, y_{2 n+1}\right), d\left(y_{2 n+1}, y_{2 n+2}\right)\right\}
$$

$$
\psi\left(d\left(y_{2 n+1}, y_{2 n+2}\right)\right) \leq \alpha \psi\left(\max \left\{d\left(y_{2 n}, y_{2 n+1}\right), d\left(y_{2 n+1}, y_{2 n+2}\right)\right\}\right)
$$

Now if $d\left(y_{2 n}, y_{2 n+1}\right) \leq d\left(y_{2 n+1}, y_{2 n+2}\right)$, for each positive integer $n$, then from (2.3), we have

$$
\psi\left(d\left(y_{2 n+1}, y_{2 n+2}\right)\right) \leq \alpha \psi d\left(y_{2 n+1}, y_{2 n+2}\right)
$$

which implies that $d\left(y_{2 n+1}, y_{2 n+2}\right)=0$, or $y_{2 n+1}=y_{2 n+2}$, contradicting our assumption that $y_{n} \neq y_{n+1}$, for each $n$. Therefore $d\left(y_{2 n+1}, y_{2 n+2}\right) \leq d\left(y_{2 n}, y_{2 n+1}\right)$, for all $n \geq 0$ and $\left\{d\left(y_{n}, y_{n+1}\right)\right\}$ is strictly decreasing sequence of positive numbers and therefore tends to a limit $r \geq 0$. If possible suppose $r>0$. Then for given $\eta>0$, there exists a positive integer $N$ such that for each $n \in N$, we have

$$
r \leq d\left(y_{n}, y_{n+1}\right)<r+\eta,
$$

Taking the limit $n \rightarrow \infty$, in (2.3) and using the continuity of $\psi$, we have or

$$
\psi(r) \leq \alpha \psi(r+\eta) \leq \alpha \psi(r),
$$

which is a contradiction unless $r=0$. Hence

$$
\lim _{n \rightarrow \infty} d\left(y_{n}, y_{n+1}\right)=0 .
$$

Next we show that $\left\{y_{n}\right\}$ is a Cauchy sequence. Suppose it is not, then there exists an $\varepsilon>0$ and since $\lim _{n \rightarrow \infty} d\left(y_{n}, y_{n+1}\right)=0$, there exists two sequences of positive numbers $\left\{y_{m(k)}\right\}$ and $\left\{y_{n(k)}\right\}$ such that for all positive integers $k, n(k)>m(k)>k$ and $d\left(y_{m(k)}, y_{n(k)}\right) \geq \varepsilon$. Assuming that $n(k)$ is the smallest positive integer, we get $n(k)>m(k)>k$,

$$
d\left(y_{m(k)}, y_{n(k)}\right) \geq \varepsilon \text { and } d\left(y_{m(k)}, y_{n(k)-1}\right)<\varepsilon .
$$

Now,

$$
\varepsilon \leq d\left(y_{m(k)}, y_{n(k)}\right) \leq d\left(y_{m(k)}, y_{n(k)-1}\right)+d\left(y_{n(k)-1}, y_{n(k)}\right)
$$

i.e.

$$
\varepsilon \leq d\left(y_{m(k)}, y_{n(k)}\right) \leq \varepsilon+d\left(y_{n(k)-1}, y_{n(k)}\right)
$$

Taking the limit as $k \rightarrow \infty$ in (2.6) and using (2.5), we have

$$
\lim _{k \rightarrow \infty} d\left(y_{m(k)}, y_{n(k)}\right)=\varepsilon .
$$

Again

$$
\begin{aligned}
d\left(y_{m(k)}, y_{n(k)}\right) \leq & d\left(y_{m k}, y_{m(k)+1}\right)+d\left(y_{m(k)+1}, y_{n(k)+1}\right) \\
& +d\left(y_{n(k)+1}, y_{n(k)}\right)
\end{aligned}
$$

and

$$
\begin{aligned}
d\left(y_{m(k)+1}, y_{n(k)+1}\right) \leq & d\left(y_{m(k)+1}, y_{m(k)}\right)+d\left(y_{m(k)}, y_{n(k)}\right) \\
& +d\left(y_{n(k)}, y_{n(k)+1}\right)
\end{aligned}
$$

Taking the limit as $k \rightarrow \infty$ and using (2.6) and (2.7), we have

$$
\lim _{k \rightarrow \infty} d\left(y_{m(k)+1}, y_{n(k)+1}\right)=\varepsilon .
$$

Again we have 


$$
d\left(y_{m(k)}, y_{n(k)}\right) \leq d\left(y_{m(k)}, y_{n(k)+1}\right)+d\left(y_{n(k)+1}, y_{n(k)}\right)
$$

and

$$
d\left(y_{m(k)}, y_{n(k)+1}\right) \leq d\left(y_{m(k)}, y_{n(k)}\right)+d\left(y_{n(k)}, y_{n(k)+1}\right) .
$$

Letting $k \rightarrow \infty$ and using (2.6) and (2.7), we have

$$
\lim _{k \rightarrow \infty} d\left(y_{m(k)}, y_{n(k)+1}\right)=\varepsilon
$$

Similarly, we have $\lim _{k \rightarrow \infty} d\left(y_{n(k)}, y_{m(k)+1}\right)=\varepsilon$.

For each positive integer $k, y_{m(k)}$ and $y_{n(k)}$ are comparable. Now using the monotone property of $\psi$ in 4), we have

$$
\begin{aligned}
& \psi\left(d\left(y_{m(k)+1}, y_{n(k)+1}\right)\right)=\psi\left(d\left(F y_{m(k)}, G y_{n(k)}\right)\right) \\
& \leq \alpha \psi\left(\max \left\{d\left(I y_{m(k)}, J y_{n(k)}\right), D\left(I y_{m(k)}, F y_{m(k)}\right), D\left(J y_{n(k)}, G y_{n(k)}\right), \frac{D\left(I y_{m(k)}, G y_{n(k)}\right)+D\left(I y_{n(k)}, F y_{m(k)}\right)}{2}\right\}\right) \\
& \leq \alpha \psi\left(\max \left\{d\left(y_{m(k)}, y_{n(k)}\right), d\left(y_{m(k)}, y_{m(k)+1}\right), d\left(y_{n(k)}, y_{n(k)+1}\right) \frac{d\left(y_{m(k)}, y_{n(k)+1}\right)+d\left(y_{n(k)}, y_{m(k)+1}\right)}{2}\right\}\right)
\end{aligned}
$$

Letting $k \rightarrow \infty$ and using (2.6)-(2.9), and the continuity of $\psi$, we have $\psi(r) \leq \alpha \psi(r)$, which is a contradiction by virtue of property of $\psi$. Therefore $\left\{y_{n}\right\}$ and hence any subsequence thereof, is a Cauchy sequence.

Suppose $J(X)$ is complete. Since $\left\{y_{2 n+1}\right\}=\left\{J x_{2 n+1}\right\}$ is a subsequence of $\left\{y_{n}\right\}$, by the above $\left\{J x_{2 n+1}\right\}$ is Cauchy and $J x_{2 n+1} \rightarrow p=J v$, for some $v \in X$.

We now show $I x_{2 n+2} \rightarrow p$. For suppose $I x_{2 n+2} \rightarrow q$.
Since $y_{2 n+1}=J x_{2 n+1} \rightarrow p$ and $y_{2 n+2}=I x_{2 n+2} \rightarrow q$ therefore, $d\left(y_{2 n+1}, y_{2 n+2}\right) \rightarrow d(p, q)$. But $\left\{d\left(y_{2 n+1}, y_{2 n+2}\right)\right\}$ is a subsequence of the strictly decreasing sequence $\left\{d\left(y_{n}, y_{n+1}\right)\right\}$ which tends to the $\lim r=0$. Therefore $\left\{d\left(y_{2 n+1}, y_{2 n+2}\right)\right\}$ tends to limit $r=0$ and hence $d(q, p)=0$ implying $q=p$. Thus $I x_{2 n+2} \rightarrow p$. Now using $(d)$, we have

$$
\begin{aligned}
& \psi\left(d\left(F x_{2 n}, p\right)\right)=\psi\left(d\left(F x_{2 n}, I x_{2 n+2}\right)\right)=\psi\left(d\left(F x_{2 n}, G x_{2 n+1}\right)\right) \\
& \leq \alpha \psi\left(\max \left\{d\left(I x_{2 n}, J x_{2 n+1}\right), D\left(I x_{2 n}, F x_{2 n}\right), D\left(J x_{2 n+1}, G x_{2 n+1}\right), \frac{D\left(I x_{2 n}, G x_{2 n+1}\right)+D\left(I x_{2 n+1}, F x_{2 n}\right)}{2}\right\}\right) \\
& \leq \alpha \psi\left(\max \left\{d(q, p), d\left(p, F x_{2 n}\right), d(p, q), \frac{d(p, q)+d\left(p, F x_{2 n}\right)}{2}\right\}\right),
\end{aligned}
$$

or

$$
\psi\left(\delta\left(F x_{2 n}, p\right)\right) \leq \psi\left(\delta\left(F x_{2 n}, p\right)\right),
$$

which is a contradiction. Consequently $d\left(F x_{2 n}, p\right) \rightarrow 0$

$$
\text { as } n \rightarrow \infty \text {. }
$$

In the same manner, it follows that $d\left(G x_{2 n+1}, p\right) \rightarrow 0$ as $n \rightarrow \infty$. We now show $G v=\{p\}$. For this, in view of $(d)^{\prime}$, we have

$$
\begin{aligned}
\psi\left(\delta\left(F x_{2 n}, G v\right)\right) & \leq \alpha \psi\left(\max \left\{d\left(I x_{2 n}, J v\right), D\left(I x_{2 n}, F x_{2 n}\right), D(J v, G v), \frac{D\left(I x_{2 n}, G v\right)+D\left(I v, F x_{2 n}\right)}{2}\right\}\right) \\
& \leq \alpha \psi\left(\max \left\{d(G v, p), d(p, p), d(p, G v), \frac{d(p, G v)+d(p, p)}{2}\right\}\right),
\end{aligned}
$$

implies

$$
\psi(d(p, G v)) \leq \psi d(G v, p)
$$

or

$$
\psi(d(p, G v)) \leq \psi d(G v, p)
$$

which is a contradiction. Consequently, $d(G v, p) \rightarrow 0$ as $n \rightarrow \infty$. Hence $G v=\{p\}=\{J v\}$. Since $G(X) \subset(X)$ there exists some $u \in X$ such that $G v=\{I u\}$. Hence $G v=\{J v\}=\{I u\}$. We now show $F u=\{I u\}$. For this, first we prove $I u \in F u$. Suppose $I u \notin F u$ then $D(I u, F u)>0$. Then in accordance with $(d)$ such that 


$$
\begin{aligned}
\psi(d(F u, I u)) & =\psi(d(F u, G v)) \leq \alpha \psi\left(\max \left\{d(I u, J v), D(I u, F u), D(J v, G v), \frac{D(I u, G v)+D(I v, F u)}{2}\right\}\right) \\
& =\alpha \psi\left(\max \left\{0, D(I u, F u), 0, \frac{0+D(I v, F u)}{2}\right\}\right) .
\end{aligned}
$$

implies $\psi(d(F u, I u)) \leq \alpha \psi D(I u, F u)$, while $D(F u, I u) \leq d(F u, I u)$. Therefore a contradiction arises. Hence $I u \in F u$. But then $M(u, v)=0$, which, by $(d)^{\prime}$, implies $\operatorname{diam}(F u)=\operatorname{diam}(G v)=\operatorname{diam}\{I u\}=0$.

Therefore $F u$ is a singleton. Since $I u \in F u$ and $F u$ is a singleton, $F u=\{I u\}$. Hence

$$
G v=\{J v\}=F u=\{I u\}=\{p\}
$$

Since the pair $\{F, I\}$ and $\{G, J\}$ are weakly com- patible,

and

$$
F p=F I u=\{I F u\}=\{I p\}
$$

$$
G p=G J u=\{J G u\}=\{J p\} .
$$

From the above, it is clear that $F p$ and $G p$ are singletons and $d(F p, G p)=d(I p, J p)$.

We now show that $I p=J p$. For instance, suppose $I p \neq J p$ then from $(d)$, we have

$$
\begin{aligned}
\psi(d(I p, J p)) & =\psi(d(F p, G p)) \leq \alpha \psi\left(\max \left\{d(I p, J p), D(I p, F p), D(J p, G p), \frac{D(I p, G p)+D(I p, F p)}{2}\right\}\right) \\
& =\alpha \psi\left(\max \left\{d(I p, J p), 0,0, \frac{d(I p, J p)+0}{2}\right\}\right)
\end{aligned}
$$

Implies as above $d(I p, J p) \rightarrow 0$ as $n \rightarrow \infty$. Hence $I p=J p$ and therefore $F p=G p=\{I p\}=\{J p\}$.
We now show $F p=\{p\}$. For, suppose $F p \neq\{p\}$. For this let $F p \neq G v$ in $(d)$, we have

$$
\begin{aligned}
\psi(d(F p, G v)) & \leq \alpha \psi\left(\max \left\{d(I p, J v), D(I p, F p), D(J v, G v) \frac{D(I p, G v)+D(I v, F p)}{2}\right\}\right) \\
& =\alpha \psi\left(\max \left\{d(F p, G v), D(F p, F p), D(G v, G v), \frac{d(F p, G v)+d(F v, F p)}{2}\right\}\right),
\end{aligned}
$$

or

$$
F p=G p=\{I p\}=\{J p\}=\{p\} .
$$

$$
\psi(d(F p, G v)) \leq \alpha \psi d(F p, G v) \leq \alpha \psi d(F p, G v), \quad \text { Let } q \in X \text { be any point satisfying }
$$

which is a contradiction. Consequently $d(F p, G v) \rightarrow 0$ as $n \rightarrow \infty$. Therefore $F p=G v=\{p\}$ and hence

$$
F q=G q=\{I q\}=\{J q\}=\{q\} .
$$

Suppose $q \neq p$ then from $(d)$, we have

$$
\psi(d(p, q))=\psi(d(F p, G q)) \leq \alpha \psi\left(\max \left\{d(I p, J q), D(I p, F p), D(J q, G q), \frac{D(I p, G q)+D(I q, F p)}{2}\right\}\right),
$$

in view of $\psi, d(p, q) \rightarrow 0$ as $n \rightarrow \infty$. Hence $q=p$.

Corollary 2.1. Let $I$ be a self mapping of a metric space $(X, d)$ and $f: X \rightarrow B(X)$ a set valued mapping satisfying

1)' $f(X) \subset(X)$,

2)' $\{f, I\}$ are weakly compatible,

$$
\text { 3)' } \psi(d(f x, f y)) \leq \alpha \psi\left(\max \left\{d(I x, I y), D(I x, f x), D(I y, f y), \frac{D(I x, f y)+D(I y, f x)}{2}\right\}\right),
$$

for all comparable $x, y \in X$, where $0<\alpha<1$ and $\psi$ is an altering distance function. If $I(X)$ is complete subspace of $X$, there exists a unique point $p \in X$ such that $f p=\{I p\}=\{p\}$. 
Proof: Taking $I=J$ and $F=G=f$ in Theorem 2.2.

Taking $I=$ identity mapping in Corollary 2.1, we get the new corollary as follows:
Corollary 2.2. Let $(X, d)$ be a complete metric space and $f: X \rightarrow B(X)$ a set valued mapping satisfying

$$
\psi(d(f x, f y)) \leq \alpha \psi\left(\max \left\{d(x, y), D(x, f x), D(y, f y), \frac{D(x, f y)+D(y, f x)}{2}\right\}\right)
$$

Then $f$ has a unique fixed point in $X$.

Proof. Obvious.

Corollary 2.3. Let $(X, \preccurlyeq)$ be a partially ordered set and suppose that there exists a metric $d$ on $X$ such that $(X, d)$ is a complete metric space. Let $I, J: X \rightarrow X$ be single valued and $F, G: X \rightarrow C B(X)$ be multival- ued mappings such that the following conditions are satisfied:

1)" $\bigcup F(X) \prec_{1} J(X)$ and $\bigcup G(X) \prec_{1} I(X)$,

2)" $\{F, I\}$ and $\{G, J\}$ are weakly compatible,

3)" if $x_{n} \rightarrow x$ is a strictly decreasing sequence in $X$, then $x_{n} \preccurlyeq x$, for all $n$,

$$
\text { 4)" } \psi(d(F x, G y)) \leq \alpha \psi\left(\max \left\{d(I x, J y), D(I x, F x), D(J y, G y), \frac{D(I x, G y)+D(I x, F y)}{2}\right\}\right) \text {, }
$$

for all comparable $x, y \in X, \operatorname{diam} F x=\operatorname{diam} G y$, where $0<\alpha<1$ and $\psi$ is an Altering distance function and suppose that one of $I(X)$ or $J(X)$ is complete. Then there exists a unique point $p \in X$ such that

$$
F p=G p=\{I p\}=\{J p\}=\{p\}
$$

Example 2.1. Let $X=\left\{(0,0),\left(0,-\frac{1}{3}\right),\left(-\frac{1}{6}, 0\right)\right\}$ be a sub set of $R^{2}$ with the order $\preccurlyeq$ defined as for

$$
\left(x_{1}, y_{1}\right),\left(x_{2}, y_{2}\right) \in X,\left(x_{1}, y_{1}\right) \preccurlyeq\left(x_{2}, y_{2}\right)
$$

if and only if $x_{1} \leq y_{1}, x_{2} \leq y_{2}$. Let $d: X \times X \rightarrow R$ be given as

$$
d(x, y)=\max \left\{\left|x_{1}-x_{2}\right|,\left|y_{1}-y_{2}\right|\right\}
$$

for $x=\left(x_{1}, y_{1}\right), y=\left(x_{2}, y_{2}\right) \in X$.

The $(X, d)$ is a complete metric space with the required properties of Theorem 2.2.
Let $I, J: X \rightarrow X$ and $F, G: X \rightarrow C B X$, be defined as follows:

$$
\begin{aligned}
& I x=x, J x=x / 2, \\
& F x=G x= \begin{cases}\{(0,0)\}, & \text { if } x=(0,0), \\
\left\{(0,0),\left(-\frac{1}{6}, 0\right)\right\}, & \text { if } x=\left(0,-\frac{1}{3}\right), \\
\{(0,0)\}, & \text { if } x=\left(-\frac{1}{6}, 0\right),\end{cases}
\end{aligned}
$$

Let $\psi:[0, \infty) \rightarrow[0, \infty)$ defined as $\psi(t)=t^{2}$, and $\alpha=1 / 2$. Then all the conditions in the Theorem 2.2 satisfied. Without loss of generality, we assume that $x \preccurlyeq y$, we discuss the following cases.

1) If $x=\left(0,-\frac{1}{3}\right), y=(0,0)$, then $\delta(F x, G y)=-\frac{1}{6}$ and

$$
\max \left\{d(I x, J y), D(I x, F x), D(J y, G y), \frac{D(I x, G y)+D(I y, F x)}{2}\right\}=\frac{1}{3}
$$

2) If $x=\left(-\frac{1}{6}, 0\right), y=(0,0)$, then $\delta(F x, G y)=0$, and

$$
\max \left\{d(I x, J y), D(I x, F x), D(J y, G y), \frac{D(I x, G y)+D(I y, F x)}{2}\right\}=\frac{1}{6}
$$

3) If $x=(0,0), y=(0,0)$, then $\delta(F x, G y)=0$, and

$$
\max \left\{d(I x, J y), D(I x, F x), D(J y, G y), \frac{D(I x, G y)+D(I y, F x)}{2}\right\}=0
$$

4) If $x=\left(-\frac{1}{6}, 0\right), y=\left(-\frac{1}{6}, 0\right)$, then $\delta(F x, G y)=0$, and 


$$
\max \left\{d(I x, J y), D(I x, F x), D(J y, G y), \frac{D(I x, G y)+D(I y, F x)}{2}\right\}=\frac{1}{6}
$$

5) If $x=\left(0,-\frac{1}{3}\right), y=\left(0,-\frac{1}{3}\right)$, then $\delta(F x, G y)=-\frac{1}{6}$ and

$$
\max \left\{d(I x, J y), D(I x, F x), D(J y, G y), \frac{D(I x, G y)+D(I y, F x)}{2}\right\}=\frac{1}{3}
$$

In all above cases, it is clearly shown that

$$
\psi(d(F x, G y)) \leq \alpha \psi\left(\max \left\{d(I x, J y), D(I x, F x), D(J y, G y), \frac{D(I x, G y)+D(I y, F x)}{2}\right\}\right),
$$

Hence the conditions of Theorem 2.2 are satisfied and shown that $\{(0,0)\}$ is a fixed point of $I, J, F$, and $G$.

\section{Acknowledgements}

Dedicated to Professor H. M. Srivastava on his $71^{\text {st }}$ Birth Anniversary.

\section{REFERENCES}

[1] R. A. Rashwan and M. A. Ahmed, "Common Fixed Points for $\delta$-Compatible Mappings,” Southwest Journal of Pure and Applied Mathematics, Vol. 1, 1996, pp. 1-6.

[2] M. A. Ahmed, "Common Fixed Point Theorems for Weakly Compatible Mappings," Rocky Mountain Journal of Mathematics, Vol. 33, No. 4, 2003, pp. 1189-1203. doi:10.1216/rmjm/1181075457

[3] B. Fisher, "Common Fixed Point of Four Mappings," Bulletin of the Institute of Mathematics, Academia Sinica, Vol. 11, 1983, pp. 103-113.

[4] B. Fisher, "Common Fixed Point Theorems for Commuting Mappings," Bulletin of the Institute of Mathematics, Academia Sinica, Vol. 9, 1981, pp. 399-406.

[5] B. Fisher, "Common Fixed Points of Mappings and Set Valued Mappings,” Rostocker Mathematisches Kolloquium, Vol. 18, 1981, pp. 69-77.

[6] B. Fisher and S. Sessa, "Two Common Fixed Point Theorems for Weakly Commuting Mappings," Periodica Mathematica Hungarica, Vol. 20, No. 3, 1989, pp. 207-218. doi:10.1007/BF01848124

[7] S. V. R. Naidu, "Some Fixed Point Theorems in Metric Spaces by Altering Distances," Czechoslovak Mathematical Journal, Vol. 53, No. 1, 2003, pp. 205-212. doi:10.1023/A:1022991929004

[8] R. A. Rashwan and M. A. Ahmed, "Fixed Points of Single and Set Valued Mappings,” Kyungpongk Mathematica Journal, Vol. 38, No. 1, 1998, pp. 29-37.
[9] G. Jungck and B. E. Rhoades, "Fixed Points for Set Valued Functions without Continuity,” Indian Journal of Pure Applied Mathematics, Vol. 29, No. 3, 1998, pp. 227-238.

[10] G. Jungck and B. E. Rhoades, "Some Fixed Point Theorems for Compatible Maps,” International Journal of Mathematics and Mathematical Sciences, Vol. 16, No. 3, 1993, pp. 417-428. doi:10.1155/S0161171293000535

[11] I. Beg and A. R. Butt, "Common Fixed Point for Generalized Set Valued Contractions Satisfying an Implicit Relation in Partially Ordered Metric Spaces,” Mathematical Communications, Vol. 15, No. 1, 2010, pp. 65-76.

[12] M. S. Khan, M. Swaleh and S. Sessa, "Fixed Points Theorems by Altering Distances between the Points," Bulletin Australian Mathematical Society, Vol. 30, No. 1, 1984, pp. 1-9. doi:10.1017/S0004972700001659

[13] S. V. R. Naidu, "Some Fixed Point Theorems in Metric Spaces by Altering Distances," Czechoslovak Mathematical Journal, Vol. 53, No. 1, 2003, pp. 205-212. doi:10.1023/A:1022991929004

[14] B. S. Choudhury, “A Common Unique Fixed Point Result in Metric Spaces Involving Generalised Altering Distances," Mathematical Communications, Vol. 10, No. 2, 2005, pp. 105-110.

[15] B. S. Choudhury and P. N. Dutta, “Common Fixed Points for Fuzzy Mappings Using Generalized Altering Distances,” Soochow Journal of Mathematics, Vol. 31, No. 1, 2005, pp. 71-81.

[16] K. P. R. Sastry and G. V. R Babu, "Some Fixed Point Theorems by Altering Distances between the Points," Indian Journal of Pure and Applied Mathematics, Vol. 30, No. 6, 1999, pp. 641-647.

[17] B. S. Choudhury and N. Metiya, "Multivalued and Singlevalued Fixed Point Results in Partially Ordered Metric Spaces,” Arab Journal of Mathematical Sciences, Vol. 17, No. 2, 2011, pp. 135-151. doi:10.1016/j.ajmsc.2011.03.001 